This is the accepted manuscript of the paper published in: IEEE JPV (Volume: 7, Issue: 1, Jan. 2017), doi: 10.1109/JPHOTOV.2016.2615680

(C) 2017 IEEE. Personal use of this material is permitted. Permission from IEEE must be obtained for all other uses, in any current or future media, including reprinting/republishing this material for advertising or promotional purposes, creating new collective works, for resale or redistribution to servers or lists, or reuse of any copyrighted component of this work in other works

\title{
Analysis of Local Minority Carrier Diffusion Lengths in Liquid Phase Crystallized Silicon Thin-Film Solar Cells
}

\author{
Paul Sonntag ${ }^{1}$, Matevž Bokalič², Miha Filipič², Tim Frijnts ${ }^{3}$, Daniel Amkreutz ${ }^{1}$, Marko Topič² and Bernd \\ $\operatorname{Rech}^{1}$ \\ ${ }^{1}$ Institut für Silizium Photovoltaik, Helmholtz Zentrum Berlin für Materialen und Energie GmbH, 12489 \\ Berlin, Germany \\ ${ }^{2}$ Faculty of Electrical Engineering, University of Ljubljana, 1000 Ljubljana, Slovenia \\ ${ }^{3}$ PVcomB, Helmholtz Zentrum Berlin für Materialen und Energie GmbH, 12489 Berlin, Germany
}

\begin{abstract}
We developed a method to quantify the local minority carrier diffusion lengths in interdigitated back-contact solar cells having a $10 \mu \mathrm{m}$ thick liquid phase crystallized (LPC) $\mathrm{Si}$ absorber by light-beam induced current (LBIC) measurements. The method is verified by 2-D simulations of the LBIC signals using ASPIN3. The effective minority carrier diffusion lengths determined this way range between $33-44 \mu \mathrm{m}$ inside a grain, which proves that advanced cell concepts like an IBC system are well suited for the LPC absorbers. Furthermore, the method has the potential to help improving the optimization of contact system geometries and it may be used to understand the influences of different grain orientations and improve the LPC-Si absorber fabrication process.
\end{abstract}

\section{INTRODUCTION}

Liquid Phase Crystallization (LPC) of Si absorbers on glass has established itself as an alternative technology approach to produce absorbers for solar cells of high electronic quality [1]-[3]. It has the potential to decrease material consumption avoiding kerf losses that are inherent to conventional wafer technologies [4]. Furthermore, it is possible to fabricate absorbers of any desired thickness between 5-40 $\mu \mathrm{m}$ since the glass functions as mechanical stabilizer. Open circuit voltages (Voc) of up to $656 \mathrm{mV}$ [3], short circuit current densities $\left(J_{S C}\right)$ of over $30 \mathrm{~mA} / \mathrm{cm}^{2}$ [5] and efficiencies up to $12.1 \%$ [6] have been realized on absorbers of $10 \mu \mathrm{m}$ thickness. To further improve device performance detailed characterization of bulk and interfaces (especially the substrate-absorber interface) is necessary. However, many of the established characterization techniques like QSSPC, TrPCD do not provide realistic results for LPC-Si absorbers, since the bulk volume is too small to provide sufficient signal.

So far the following approaches have been tried to estimate bulk lifetime, glass side surface recombination velocities and thus minority carrier diffusion lengths of LPC-Si absorbers: 1-D QE and $\mathrm{j}-\mathrm{V}$ simulations using AFORS-HET [3], evaluating plots of inverse IQE as function of absorption depth [6]-[8], depth resolved EBIC [9], transient PL [10] and high-frequency capacitance voltage $(\mathrm{C}-\mathrm{V})$ measurements [11]. However, only single-sided contact systems exist on LPC-Si absorbers which show a 2-D current flow pattern [5], [12], so 1-D simulations might not suffice to adequately represent the cell. Using inverse IQE plots, only a small wavelength window between $450-600 \mathrm{~nm}$ can be evaluated, since outside of this regime there is an uncertain amount of parasitic absorption that is hard to determine influencing the evaluation. Transient PL has the advantage that it can resolve the signal spatially but has so far not been tested on advanced contact systems. C-V measurements need careful sample preparation and cannot be applied on the finished device. In this work, we measured light-beam induced current (LBIC) signal drops over the absorber contact in LPC-Si solar cells with an IBC system. From these drops the local minority charge carrier diffusion length $\left(L_{\text {diff }}\right)$ is determined. The use of this approach is validated by 2-D simulations using ASPIN3. As enhancement to the conference proceedings [13], an additional simulation study was performed to differentiate between interface (surface recombination) and bulk (lifetime) effects influencing the effective diffusion length $L_{\text {diff. }}$.

\section{EXPERIMENTAL AND SIMULATIONS}

\section{A. Absorber and contact system preparation}

A passivating $\mathrm{SiO}_{x} / \mathrm{SiN}_{\mathrm{x}} / \mathrm{SiO}_{\mathrm{x}}$ interlayer stack was deposited on a glass substrate prior to evaporating a $10 \mu \mathrm{m}$ thick silicon absorber. Details on the interlayer setup and development can be found in [11], [14], [15]. The $10 \mu \mathrm{m}$ thick Si on glass was crystallized using an electron beam to form elongated grains [3]. The grains have the same height as the absorber, are up to few $\mathrm{mm}$ in width and up to $\mathrm{cm}$ in length [16]. The absorber doping was n-type with a concentration of $\sim 5 \times 10^{16} / \mathrm{cm}^{3}$. After standard $\mathrm{KOH}$-texturing, a contact system with an area of $1 \mathrm{x} 1 \mathrm{~cm}^{2}$ was processed according to the steps described in [5]. It is schematically depicted in Fig. 1. Interdigitated regions of 
This is the accepted manuscript of the paper published in: IEEE JPV (Volume: 7, Issue: 1, Jan. 2017), doi: 10.1109/JPHOTOV.2016.2615680

$\mathrm{n}$ and $\mathrm{i} / \mathrm{p}$ amorphous hydrogenated Si were structured using an alkaline selective etching process described in [5], [17]. The metallization of the cell is comprised of sputtered ITO and Ag. The steps were all conducted at the Helmholtz-Zentrum Berlin. The finished cell was state-of-the-art with a $V_{O C}>610 \mathrm{mV}$ and $J_{S C}$ close to $30 \mathrm{~mA} / \mathrm{cm}^{2}$.

\section{B. LBIC measurements}

LBIC measurements were conducted using a self-built setup at the University of Ljubljana with a laser of $638 \mathrm{~nm}$ wavelength (corresponding to an absorption depth in $\mathrm{Si}$ of $3.3 \mu \mathrm{m}$ ), a spot size below $10 \mu \mathrm{m}$ and an $\mathrm{x}, \mathrm{y}, \mathrm{z}$ stage with minimum step width of $1 \mu \mathrm{m}$ [18].

\section{Simulations}

Simulations were performed using ASPIN3, a numerical 2-D solar cell simulation software, developed at the University of Ljubljana which is able to calculate 1-D LBIC profiles [19].

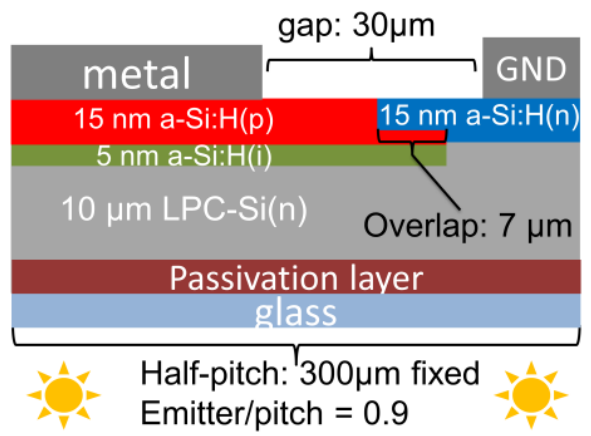

Fig. 1. Schematic of the IBC unit cell used for simulation. Simulation domain is rectangular. Passivaton layer and metal electrodes are implemented as boundary conditions for electrical calculation, but fully included in the optical calculation of the generation rate.

\section{RESULTS}

\section{A. Simulations}

A simulation structure based on the geometry of the investigated solar cell was implemented into ASPIN3. An overview of the parameters is shown in Fig. 1. LBIC line scans over the unit cell (half pitch) were simulated. To calculate the optical parameters the PVlighthouse software wafer ray tracer[20] and $(n, k)$ data from [21]-[23] was used. For the electrical properties of the a-Si:H layers the values were taken from [19]. For the poly-crystalline absorber, the electronic properties of mono-crystalline silicon were assumed, except for mobilities. For the latter, a reduced mobility $(80 \%$ of the mobility calculated from the Masetti Model [24]) was assumed to account for the polycrystalline structure. This value is in accordance with Hall measurements of recent LPC-Si absorbers[8], [25]. Doping density $\mathrm{N}_{\mathrm{D}}$ was
$5 \times 10^{16} / \mathrm{cm}^{3}$. The front side surface recombination velocity $(S R V)$ was modeled using $J_{n}=-q^{*} S R V^{*}\left(n-n_{0}\right)$ (and analogously for holes) and was varied along with the bulk SRH lifetime $\tau$. Auger and radiative recombination are negligible as SRH recombination is dominant [4]. The backsided SRV was kept constant and is only being influenced by the low lifetimes and mobilities in the a-Si layers. The influence is negligible, due to the small layer thickness of 15$20 \mathrm{~nm}$. Fig. 2 shows exemplarily simulated LBIC signals with absorbers of various $\tau$ and $S R V$ that are in the range of values estimated from previous works [3], [8].

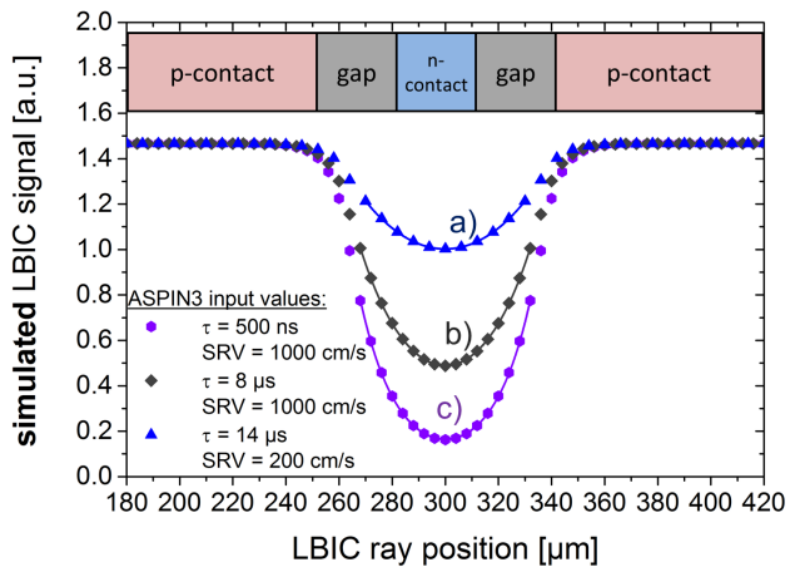

Fig. 2. Simulated LBIC line scans over the full absorber contact of the structure shown in Fig. 1. The different areas of the cell are depicted in the boxes on top. Dots: Simulated LBIC signal points with different front surface recombination velocities $S R V$ and bulk lifetimes $\tau$ listed in the inset. Lines: Double exponential fits according to equation (3).

Below the absorber $\mathrm{n}$-contact is a distinct drop in collection signal since minority carriers have to travel laterally to the emitter which enhances recombination probability. The characteristic parameter to describe this recombination pathway is the effective diffusion length $L_{d i f f}$. The latter takes bulk lifetime and $S R V$ into account can be calculated from the ASPIN3 input parameters to

$$
L_{\text {diff }}=\sqrt{\frac{k T}{e} \cdot \mu \cdot \tau_{\text {eff }}} \quad,
$$

with the Boltzman constant $k$, absolute temperature $T$, elementary charge $e$, minority carrier mobility $\mu$, and the effective lifetime of

$$
\tau_{\text {eff }}=\left(\frac{1}{\tau}+\frac{S R V}{H}\right)^{-1},
$$

$\tau$ being the SRH bulk minority lifetime, and $H$ the absorber height. Equation (2) is a special case of $\tau$ eff described in[26] that can only be applied for absorbers with a negligible backside SRV and considering thin $(<50 \mu \mathrm{m})$ absorbers. 
This is the accepted manuscript of the paper published in: IEEE JPV (Volume: 7, Issue: 1, Jan. 2017), doi: 10.1109/JPHOTOV.2016.2615680

Another way to determine the diffusion length is by fitting the simulated data from Fig. 2. According to Green [27], assuming a point-like generation rate that is zero everywhere else, the collection probability decreases exponentially from the source of the generation. This means in the case of an IBC system the collection probability $p(x)$ will increase exponentially from the middle of the absorber contact to a maximum plateau value below the emitter contacts. Hence, $p(x)$ can be expressed around the middle of the absorber contact as the sum of an exponential decay and growth

$$
p(x)=\left(e^{\frac{-\left(x-x_{1}\right)}{L_{d i f f}}}+e^{\frac{\left(x-x_{2}\right)}{L_{d i f f}}}\right)
$$

The parameters $x_{1}$ and $x_{2}$ describe the shift in $\mathrm{x}$-axis position ( $\mathrm{x}$-axis being the LBIC scan direction) of the exponential functions relative to each other according to the contact geometries. I.e. $\left(x_{2}-x_{1}\right)$ can be interpreted as the BSF width; $L_{\text {diff }}$ is the effective minority carrier diffusion length.

In Table I the values for $L_{\text {diff }}$ from the ASPIN3 input parameters calculated via the equations (1) and (2) and the results of the fits using equation (3) for the examples of Fig. 2 are listed. As can be seen, the values correspond to each other very well. This is not evident, since equation (3) is only valid for a one-dimensional case. The slight difference stems from the finite absorber height which causes a minor 2-D charge flow instead of 1-D for an infinitely thin absorber. This is also why the edges at the beginnings of the emitter contacts are smoothed out and the fit has to be started a little bit later. Nevertheless, the values correspond to each other well within a wide range of $S R V$ and $\tau$ values for the given absorber thickness of $10 \mu \mathrm{m}$. Once Ldiff is lower than the absorber thickness the 2-D component in the charge flow becomes more prevalent and the fit result and the values calculated from ASPIN3 input parameters do not correspond well to each other anymore (not shown).

\section{B. LBIC measurements}

A 2-D LBIC overview of the solar cell was recorded and is depicted in Fig 3. Darker shades represent areas with low current signal which corresponds to a low collection probability. The image shows clearly the poly-crystalline nature of LPC-Si material. Overlain to this irregular grain structure is the regular pattern of the contact grid. The absorber contacts ( $30 \mu \mathrm{m}$ in width), appear as strips of low collection in the image. The overview image was used as a reference to record several more detailed regions with a step width of $5 \mu \mathrm{m}$ and a beam diameter below $10 \mu \mathrm{m}$. One of these detailed regions is displayed exemplarily in the inset of the figure. Two regions inside grains with low dislocation density are marked therein by dotted lines (1 and 2). Two other areas are marked crossing or directly on crystal faults (3 and 4). The areas were chosen such that areas of high and low collection signal (corresponding to high and low absorber quality) are included.

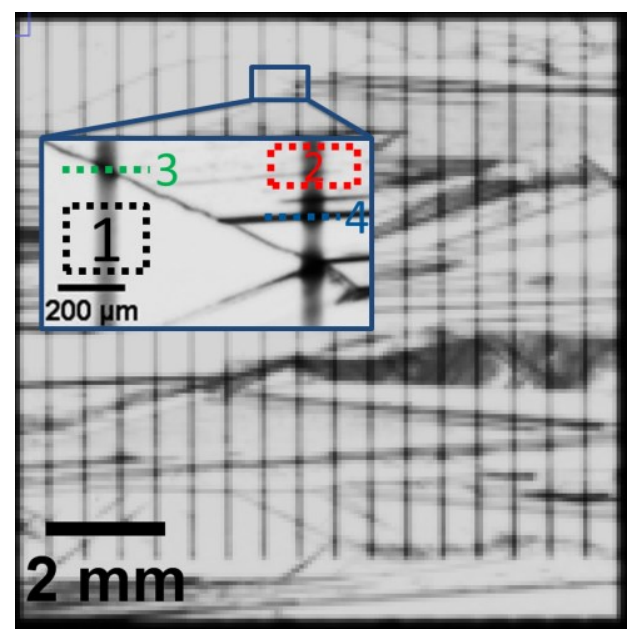

Fig. 3. 2-D LBIC scan of IBC-SHJ solar cell on a $10 \mu \mathrm{m} \mathrm{LPC-Si}$ absorber on glass. The overview serves as a reference for the more

TABLE I

COMPARISON OF $L_{\text {DIFF }}$ FROM ASPIN3 INPUT PARAMETERS AND EXPONENTIAL FITS

\begin{tabular}{|l|c|c|c|c|}
\hline & $\tau / \mu \mathrm{s}$ & $S R V / \mathrm{cm} / \mathrm{s}$ & ASPIN3 $/ \mu \mathrm{m}$ & Fit $/ \mu \mathrm{m}$ \\
\hline a) & 14 & 200 & 46.9 & 47.1 \\
\hline b) & 8 & 1000 & 23.0 & 23.7 \\
\hline c) & 0.5 & 1000 & 14.1 & 14.3 \\
\hline
\end{tabular}

detailed scan that is zoomed out in the blue frame. Four areas of different absorber quality underneath the absorber contact are marked.

Averaged LBIC line-scans were plotted from these areas and are displayed in Fig. 4. There is a distinct drop at the absorber contact that has already been described in [5] and [6] and shows the same distinct features as in the simulations. Fits using equation (3) were performed on the four exemplary linescans and are also shown in Fig. 4. The fit yielded a diffusion length of $44,33,19$, and $12 \mu \mathrm{m}$ for areas $1,2,3$ and 4 , respectively. The quality is as good as for the fits for the simulated curves in Fig. 2. Hence, we conclude that the simulation represents the behavior of the real device very well and that the method to extract diffusion lengths via fitting the signal drop over the absorber contact is verified. For the highly dislocated area 4 the result should be regarded with care as the result is already in the order of the absorber thickness. General sources of error aside from the error of the fit itself (which is negligible) are the finite beam size of the LBIC beam and fluctuations of the $x, y$ stage stepper motor and the measured signal. The former error source was estimated by deconvolution of the beam, the latter was actually accounted for in averaging the signals. As an upper estimate we suggest the method can be as accurate as $\pm 5 \mu \mathrm{m}$. 
This is the accepted manuscript of the paper published in: IEEE JPV (Volume: 7, Issue: 1, Jan. 2017), doi: 10.1109/JPHOTOV.2016.2615680

We see a wide spread in the resulting diffusion lengths between areas with low dislocation/fault density and higher one. This underlines the necessity for large grains and low defect absorbers or grain boundaries and dislocations that are less electrically active (better passivated) to reach higher diffusion lengths and thus also better solar cell performance.

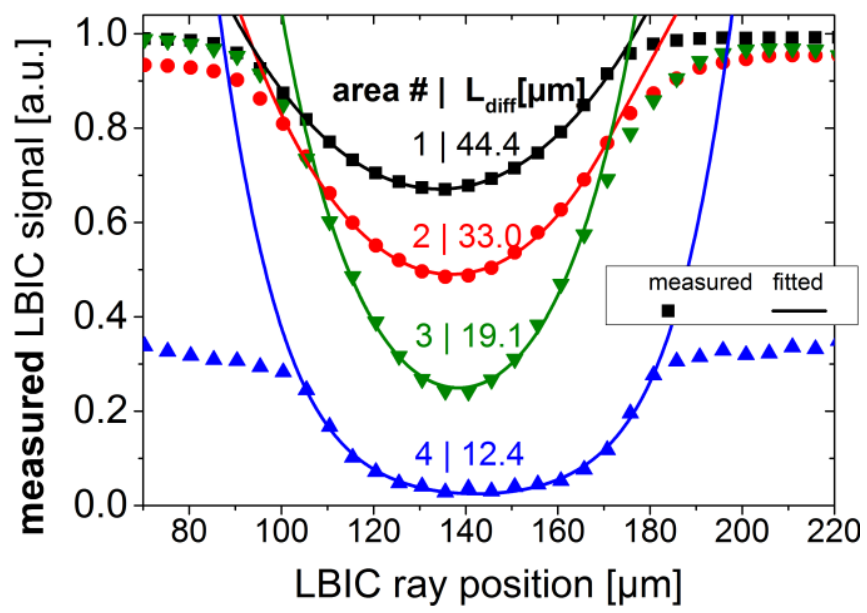

Fig. 4. Dots: Measured and averaged LBIC data from line-scans over the absorber/BSF contact from the areas highlighted in Fig.3. Lines: Double exponential fits according to equation (3).

\section{Estimating $S R V$ and $\tau$}

From both simulated as well as measured LBIC profiles the ratio between the minimum signal in the middle of the absorber contact (minimum LBIC collection) and the plateau under the emitter contact (maximum collection) can be calculated. We suggest this collection efficiency ratio as a possible figure of merit for the material quality (which includes quality of the absorber and both front and back side interfaces). Note that it is also strongly depending on the absorber contact width. To clarify, the collection efficiency ratio is graphically depicted in Fig. 5 a). In general, the closer the ratio is to 1 , the less pronounced is the signal drop, more minority charge carriers are collected from beneath the absorber contact and consequently, the better is the material quality in terms of $L$ diff and thus $S R V$ and $\tau$. Again, we are only investigating a front side $S R V$, the SRV at the back side is neglected due to the good amorphous silicon passivation properties. To investigate the influence that the front $S R V$ and $\tau$ have separately on this figure of merit several LBIC profiles were simulated and the collection efficiency was plotted against the lifetime $\tau$ in Fig. 5 b) for several $S R V$. For convenience, the bulk diffusion lengths $(\mathrm{SRV}=0)$ corresponding to the bulk lifetimes $\tau$ have been added as second $\mathrm{x}$-axis on top and also the effective diffusion lengths for $8 \mu$ s lifetime are included. The measured collection ratios of the areas inside one grain (areas 1 and 2) from Fig. 3 have been added as horizontal dashed guidelines. From Fig. 5 b) we can deduce the following: Firstly, no matter how long the
SRH bulk lifetime $\tau$ is, the $S R V$ (for our given absorber height $H=10 \mu \mathrm{m}$ and non-emitter contact width of $90 \mu \mathrm{m}$ ) will always eventually limit the collection efficiency and stay below a certain value for this given $S R V$. This underlines the importance of a well passivated LPC-Si/glass interface. Secondly, with $\tau$ below $\sim 4 \mu$ s a collection as high as for area 1 cannot be achieved even assuming a perfect front side $(S R V=0 \mathrm{~cm} / \mathrm{s})$. In conclusion we can state that $\tau$ should be longer than $\sim 4 \mu$ s for area 1 and higher than $\sim 2 \mu$ s for area 2. Thirdly, $S R V$ cannot exceed $\sim 600 \mathrm{~cm} / \mathrm{s}$, otherwise the collection will remain below the measured value for area 2 no matter how long the lifetime $\tau$ becomes. For area 1 the maximum $S R V$ has to be in the range of $\sim 200 \mathrm{~cm} / \mathrm{s}$.

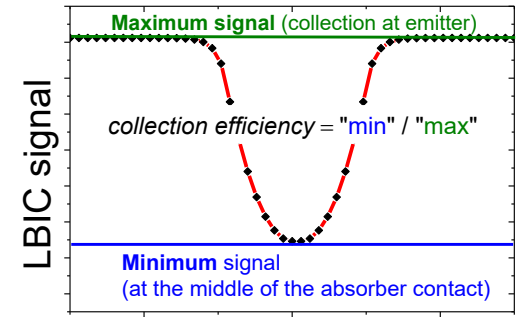

LBIC ray position

a)

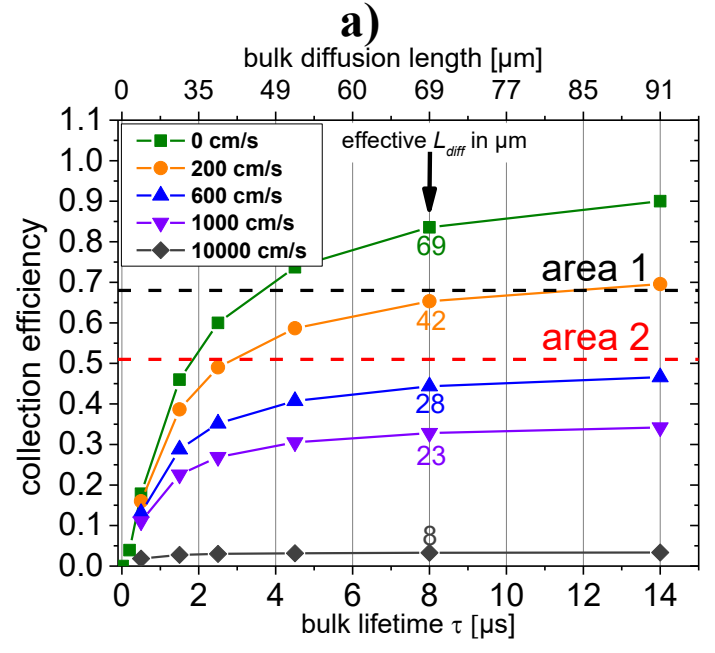

b)

Fig. 5. a) Graphical explanation of the figure of merit for the material quality. It is the ratio between maximum plateau value and the minimum LBIC current collection in the middle of the absorber contact. b) LBIC collection efficiency from simulations as a function of SRH bulk lifetime $\tau$ or bulk diffusion length for several different $S R V$ (lines are guides to the eye). The collection efficiency of measured data from area 1 and 2 are implemented as horizontal dashed guidelines. The bulk diffusion length corresponding to the lifetimes is added as upper $\mathrm{x}$-axis. The diffusion lengths corresponding to the values at $8 \mu$ s were also implemented for convenience.

\section{DISCUSSION}

In this work we developed a model to fit effective local minority carrier diffusion lengths from LBIC line scans. These 
This is the accepted manuscript of the paper published in: IEEE JPV (Volume: 7, Issue: 1, Jan. 2017), doi: 10.1109/JPHOTOV.2016.2615680

scans were performed over the absorber contact of thin-film silicon IBC solar cells on glass. The model was validated using the 2-D simulation software ASPIN3. The diffusion lengths between 33-44 $\mu \mathrm{m}$ that were found inside grains of the cell prove good material quality for thin Si absorbers on glass that can by now be realized using the liquid phase crystallization. Furthermore, the results validate the use of complex contact systems like an IBC-SHJ system since diffusion lengths are much greater than the absorber thickness. The fit procedure is independent of the IBC cell geometries and also works, if the signal drops to zero in the middle of the absorber contact (not shown). However, a sufficient signal drop is needed to use the method which might not be the case for excellently passivated front sides and bulk lifetimes exceeding $\sim 50 \mu$ s.

With the help of detailed simulations of the LBIC signals a lower limit for the bulk SRH lifetime and an upper limit for the surface recombination velocity at the LPC-Si/glass interface can be estimated. Assuming the simulations to adequately represent the experiment lifetimes are in the order of 2-4 $\mu$ s or higher, and recombination velocities around $600 \mathrm{~cm} / \mathrm{s}$ or lower. These values are in-line with results from [3], where the upper limit of the front $S R V$ was estimated to be $500 \mathrm{~cm} / \mathrm{s}$, and the lower limit of the lifetime to be $1 \mu \mathrm{s}$. This is despite the fact that the simulations in [3] were 1-D. Gabriel et al.[8] and Kühnapfel et al.[10] extracted the diffusion length of LPC-Si absorbers via inverse IQE plots and found 14 and $11 \mu \mathrm{m}$, respectively. These values are lower than the ones determined on areas $1,2,3$ by LBIC in this work. The difference may be due to various reasons. Firstly, there is an ongoing development and improvement on LPC-Si absorber fabrication, so it is difficult to compare absorbers from different stages in the development. Secondly, the diffusion lengths in this work are local ones and global values from IQE evaluation differ much from those obtained on certain spots as seen comparing the areas 1-4 in Fig. 4. Generally, these evaluation techniques become less accurate as the ratio between $L_{\text {diff }}$ and the length it is observed on decreases. For inverse IQE plots the observed length is the absorber thickness of $\sim 10 \mu \mathrm{m}$, whereas with LBIC evaluation, the whole absorber contact width serves as observed length. This makes the latter technique more accurate for higher diffusion lengths. The accuracy is also higher because it is a relative method (comparing emitter-signal with BSF-signal) which does not depend on parasitic absorption as inverse IQE evaluation.

\section{CONCLUSION}

In this work we presented a method to locally determine effective minority carrier diffusion lengths from LBIC linescans over the absorber contact of IBC cells on glass. The method can help in understanding the influences of different grain orientations (e.g. on passivation) and thus help improving the crystallization process to form liquid phase crystallized $\mathrm{Si}$ absorbers and to optimize contact grid geometries. Further studies will focus on evaluating the absorbers statistically and in more detail.

\section{ACKNOWLEDGEMENT}

The authors would like to thank Manuel Hartig, Tobias Hänel, Kerstin Jacob, Mona Wittig, Martin Muske, and Erhard Conrad at HZB, Berlin for experimental support in device fabrication and characterization and the whole LPVO group of University Ljubljana for fruitful discussions and support.

Special thanks go to the $\mathrm{HZB} \mathrm{PhD}$ abroad program and Gabriele Lampert in particular.

\section{REFERENCES}

[1] J. Dore, D. Ong, S. Varlamov, R. Egan, and M. A. Green, "Progress in Laser-Crystallized Thin-Film Polycrystalline Silicon Solar Cells: Intermediate Layers, Light Trapping, and Metallization," IEEE J. Photovolt., vol. 4, no. 1, pp. 33-39, Jan. 2014.

[2] Andrä, G., Höger, I., Bergmann, J., Gawlik, A., Plentz, J., Ose, E., and Schippel, S., "Laser crystallized silicon layers for multicrystalline thin-film solar cells," 24th Eur. Photovolt. Sol. Energy Conf., pp. 2521-2524.

[3] J. Haschke, D. Amkreutz, L. Korte, F. Ruske, and B. Rech, "Towards wafer quality crystalline silicon thin-film solar cells on glass," Sol. Energy Mater. Sol. Cells, vol. 128, pp. 190-197, Sep. 2014.

[4] J. Haschke, D. Amkreutz, and B. Rech, "Liquid phase crystallized silicon on glass: Technology, material quality and back contacted heterojunction solar cells," $J p n$. J. Appl. Phys., vol. 55, no. 4S, p. 04EA04, Mar. 2016.

[5] P. Sonntag, J. Haschke, S. Kühnapfel, T. Frijnts, D. Amkreutz, and B. Rech, "Interdigitated back-contact heterojunction solar cell concept for liquid phase crystallized thin-film silicon on glass," Prog. Photovolt. Res. Appl., Jan. 2015.

[6] T. Frijnts, S. Kühnapfel, S. Ring, O. Gabriel, S. Calnan, J. Haschke, B. Stannowski, B. Rech, and R. Schlatmann, "Analysis of photo-current potentials and losses in thin film crystalline silicon solar cells," Sol. Energy Mater. Sol. Cells, vol. 143, pp. 457-466, Dezember 2015.

[7] D. Amkreutz, J. Haschke, S. Kuhnapfel, P. Sonntag, and B. Rech, "Silicon Thin-Film Solar Cells on Glass With Open-Circuit Voltages Above $620 \mathrm{mV}$ Formed by Liquid-Phase Crystallization," IEEE J. Photovolt., vol. 4, no. 6, pp. 1496-1501, Nov. 2014.

[8] O. Gabriel, T. Frijnts, N. Preissler, D. Amkreutz, S. Calnan, S. Ring, B. Stannowski, B. Rech, and R. Schlatmann, "Crystalline silicon on glass-interface passivation and absorber material quality," Prog. Photovolt. Res. Appl., Jan. 2015.

[9] W. Seifert, D. Amkreutz, T. Arguirov, H. M. Krause, and M. Schmidt, "Analysis of Electron-Beam Crystallized Large Grained Si Films on Glass Substrate by EBIC, 
This is the accepted manuscript of the paper published in: IEEE JPV (Volume: 7, Issue: 1, Jan. 2017), doi: 10.1109/JPHOTOV.2016.2615680

EBSD and PL," Solid State Phenom., vol. 178-179, pp. 116-121, 2011.

[10] S. Kühnapfel, J. Huang, A. Teal, H. Kampwerth, D. Amkreutz, S. Gall, and S. Varlamov, "Lifetime analysis of laser crystallized silicon films on glass," J. Appl. Phys., vol. 118, no. 5, p. 55304, Aug. 2015.

[11] N. Preissler, J. A. Töfflinger, I. Shutsko, O. Gabriel, S. Calnan, B. Stannowski, B. Rech, and R. Schlatmann, "Interface passivation of liquid-phase crystallized silicon on glass studied with high-frequency capacitance-voltage measurements," Phys. Status Solidi A, Feb. 2016.

[12] J. Haschke, L. Jogschies, D. Amkreutz, L. Korte, and B. Rech, "Polycrystalline silicon heterojunction thin-film solar cells on glass exhibiting $582 \mathrm{mV}$ open-circuit voltage," Sol. Energy Mater. Sol. Cells, vol. 115, pp. $7-$ 10, Aug. 2013.

[13] P. Sonntag, B. Matevz, M. Filipic, T. Frijnts, D. Amkreutz, M. Topic, and B. Rech, "Analysis of Local Minority Carrier Diffusion Lengths in Liquid Phase Crystallized Silicon Thin-Film Solar Cells," IEEE PVSC 43 Conf. Proc., vol. accepted, Jun. 2016.

[14] J. Dore, S. Varlamov, and M. A. Green, "Intermediate Layer Development for Laser-Crystallized Thin-Film Silicon Solar Cells on Glass," IEEE J. Photovolt., vol. PP, no. 99, pp. 1-8, 2014.

[15] D. Amkreutz, W. D. Barker, S. Kuhnapfel, P. Sonntag, J. Haschke, S. Gall, J. Schmidt, O. Gabriel, and B. Rech, "Liquid phase crystallized silicon solar cells on glass: Material quality and device design," in Photovoltaic Specialist Conference (PVSC), 2015 IEEE 42nd, 2015, pp. 1-3.

[16] S. Kühnapfel, N. H. Nickel, S. Gall, M. Klaus, C. Genzel, B. Rech, and D. Amkreutz, "Preferential $\{100\}$ grain orientation in 10 micrometer-thick laser crystallized multicrystalline silicon on glass," Thin Solid Films, vol. 576, pp. 68-74, Feb. 2015.

[17] J. Nakamura, N. Asano, T. Hieda, C. Okamoto, H. Katayama, and K. Nakamura, "Development of Heterojunction Back Contact Si Solar Cells," IEEE J. Photovolt., vol. 4, no. 6, pp. 1491-1495, Nov. 2014.
[18] M. Bokalič and M. Topič, "Light beam characterisation of LBIC apparatus and selected complementary applications," in Proc. of 46th MIDEM Conference, 2010, pp. 233-237.

[19] M. Filipič, Z. C. Holman, F. Smole, S. D. Wolf, C. Ballif, and M. Topič, "Analysis of lateral transport through the inversion layer in amorphous silicon/crystalline silicon heterojunction solar cells," J. Appl. Phys., vol. 114, no. 7, p. 74504, Aug. 2013.

[20] wafer ray tracer (version 1.4.3). .

[21] Z. C. Holman, M. Filipič, A. Descoeudres, S. D. Wolf, F. Smole, M. Topič, and C. Ballif, "Infrared light management in high-efficiency silicon heterojunction and rear-passivated solar cells," J. Appl. Phys., vol. 113, no. 1, p. 13107, Jan. 2013.

[22] K. R. McIntosh, T. C. Kho, K. C. Fong, S. C. BakerFinch, Y. Wan, N. Zin, E. T. Franklin, D. Wang, M. D. Abbott, N. E. Grant, E. Wang, M. Stocks, and A. W. Blakers, "Quantifying the optical losses in back-contact solar cells," in Photovoltaic Specialist Conference (PVSC), 2014 IEEE 40th, 2014, pp. 0115-0123.

[23] S. C. Baker-Finch and K. R. McIntosh, "Reflection of normally incident light from silicon solar cells with pyramidal texture," Prog. Photovolt. Res. Appl., vol. 19, no. 4, pp. 406-416, Jun. 2011.

[24] G. Masetti, M. Severi, and S. Solmi, "Modeling of carrier mobility against carrier concentration in arsenic-, phosphorus-, and boron-doped silicon," IEEE Trans. Electron Devices, vol. 30, no. 7, pp. 764-769, Jul. 1983.

[25] S. Kühnapfel, S. Gall, B. Rech, and D. Amkreutz, "Towards monocrystalline silicon thin films grown on glass by liquid phase crystallization," Sol. Energy Mater. Sol. Cells, vol. 140, pp. 86-91, Sep. 2015.

[26] A. G. Aberle, Crystalline Silicon Solar Cells: Advanced Surface Passivation and Analysis. Centre for Photovoltaic Engineering, University of New South Wales, 1999.

[27] M. A. Green, "Solar cells: operating principles, technology, and system applications," Jan. 1982. 